Research Journal of Applied Sciences 13 (11): 663-674, 2018

ISSN: $1815-932 \mathrm{X}$

(C) Medwell Journals, 2018

\title{
Review of Intelligent Robotics Algorithms for Assistive Agents
}

\author{
Natalie Segura Velandia Ruben D. Hernandez Beleno and Robinson Jimenez Moreno \\ Faculty of Engineering, Nueva Granada Military University, Bogota, Colombia
}

\begin{abstract}
In this study it is present a review of artificial intelligence algorithms applied to robotic agents, emphasizing learning from Convolutional Neural Networks (CNN). For the construction of this review, a bibliographical research was carried out in the databases of scientific articles, research journals and technological advances where the most important works are described which implement different methods in a variety applications such as autonomous vehicles, robots for space exploration and robotic manipulators in the planning of trajectories making use of CNN. Artificial Intelligence (AI) is defined as the technique in which there are developed algorithms capable of reasoning, learning and making decisions based on experience. This technique has achieved its interaction with robotic agents getting to create what is now known as intelligent robots, capable of solving and performing multiple tasks of daily life but like all developing science it is still possible to optimize and improve their algorithms to achieve human precision, referred to the manner and efficiency with which a human develops different types of activities. As a result, some of the applications that are available to people will be presented in order to highlight the extent to which this synergy of technologies has reached its development.
\end{abstract}

Key words: Neural networks, deep learning, fuzzy logic, autonomous vehicles, artificial intelligence, robotic agents

\section{INTRODUCTION}

The beginning of what is now known as artificial intelligence was conceived in 1943 with researchers like Warren McCulloch and Walter Pitts who developed the first model of artificial neurons, proposing a mathematical model to imitate on a small scale the functioning of human brain neurons, based on activation functions, synaptic weights and inputs (McCulloch and Pitts, 1943).

Years later in 1950 Alex Turing, considered the father of artificial intelligence, raises the question "Can machines think?" which he answered by means of his ingenious Turing test which seeks to determine if the machine is intelligent or not. To develop this test it must have at least 3 participants an interrogator (human person) a machine and another person where the interrogator will ask a certain number of questions to each interviewee who are in separate spaces and their only objective is to convince the interrogator that they are human (Turing, 1950). From the above there is a growing interest in the development and research in this area and in 1956, a group of researchers and professors met in order to build a machine that was capable of performing functions from the numerical calculation and based on this achieve intelligent actions. This conference was known as the Darthmount conference where different personages participated such as Arthur Samuel, Oliver Selfridge, Ray Solomonff, Allen
Newell, Simon Hebert, John McCarthy, Marvin Minsky, Claude Shannon and Nathaniel Rochester who in this same event, define and give the name to what is now known as Artificial Intelligence (AI). After this in 1964, (Zadeh, 1965) writes an article where he defines a fuzzy set which unlike traditional sets with elements belonging or not, establishes a function that assigns to each element a degree of belonging that oscillates between zero and one, being these the bases of what is known as fuzzy logic which allows to set computational rules similar to human thought.

In 1969, it is presented the development of the Stanford arm that shows the robotic abilities reached by means of artificial vision, i.e., equipped with a camera and controlled by computer, where an experiment is developed in which the manipulator stacked blocks according to the entry criteria that were given (Scheinman, 1969). In this same year, the first bipedal robot called WAP-1 was developed, designed by Ichiro Kato who also designed an approximation of the human muscle, connecting airbags to a frame simulating an artificial muscle, thanks to this achievement, the third stage was developed which was the WAP-3, implemented to achieve reproducing the march on a flat surface and going up or down stairs (Nonami et al., 2013). On the other hand in 1986 the model proposed by Rumelhart and McClelland defines an element of process or artificial neuron as a device from a

Corresponding Author: Natalie Segura Velandia, Faculty of Engineering, Nueva Granada Military University, Bogota, Colombia 
set of inputs that generates a single output (McClelland and Rumelhart, 1986). It should be added that later in 1987, Fischels and Firschein described an intelligent agent as everything capable of perceiving their environment through sensors, processing these sensory perceptions and responding in a rational way (Stuart and Norving, 1995), i.e., correspondingly in the development of their activities by means of actuators or elements that react to a stimulus with the capacity to improve the expected result (Carolina and Lemus, 2011). After this and beginning an era of technological evolution. Krizhevsky et al. (2012) proposes a model based on CNNs that was able to classify images in 1000 categories, revolutionizing the field of computational vision. Later Krizhevsky (2014) carried out multi-GPU implementations where establishes a new way of implanting parallelisms between the formation of convolutional neural networks, enhancing their ability to process images.

The first high-impact research was developed by Simonyan and Zisserman (2014) where they presented the effect that a deep convolutional network has on the recognition of large-scale images where its implementation was based on a very deep 19-layer neural network for the classification of these images, showing successful results in classification accuracy. The second work was carried out by Levine (2016) where they carry out the implementation of a set of manipulators based on convolutional neural network training in order to recognize an object and perform the best displacement for its grip.

According to, the above and in relation to what is presented in this research it is important to define some questions such as what is a robotic agent? A robotic agent is anything capable of perceiving its environment by means of sensors (for example cameras) and interacting with it by means of actuators (motors) that will be responsible for executing an action depending on the information received by the sensor. But how does it work? A robotic agent must comply with a basic structure: behavior which is described as those actions that occur after certain perceptions a program, also known as the skeleton of the agent since, it performs the implementation of a function that transforms perception into actions (seeks answers). The robotic agent is identified by four characteristics, first it must not be totally pre-programmed it means that a program that takes away the autonomy of making decisions should not be made. Second, a cognitive architecture is provided that allows the relationship between the inputs and the actions that must be performed. Third, it must have the ability to generate a sensory map that helps it determine actions to survive. Fourth, it must achieve the desired goals. And finally what is the use of robotic agents? They may be use in the solution of high risk tasks avoiding the involvement of human intervention or simply in routine activities that over time will generate health problems and all those that allow a solution that improves the quality of life of people.

\section{MATERIALS AND METHODS}

Artificial intelligence: Artificial intelligence throughout its development has received several definitions from different points of view for example from the human act or moral judgment; "The study of how to make computers do things in which people are better at the moment" (Rich and Knight, 1991) from rational thinking: "The study of computational processes that makes it possible to perceive, reason and act" (Winston, 1992) and finally, from the rational act; "The study of the design of intelligent agents" (Poole et al., 1998). From the above it can be defined as the study of simulating superior functions of the brain from mathematical calculations that make possible the perception and reasoning of an agent that has no life.

Distributed artificial intelligence: Distributed Artificial Intelligence (DAI) arises from the development of artificial intelligence where it is not wanted to package information into a single information system but instead focus on the design and construction of agents that cooperate to solve the problem where each agent possesses their degree of intelligence and the union of all produces a total intelligence or called distributed intelligence. Its main application is in real-time environments characterized by being dynamic, decentralized and incompletely specific from which the technique is born that tries to solve problems from a distributed or collective behavior that turns out to be more efficient than individual behavior (Thrun et al., 2006). It is implemented by typical agents through social relationships as illustrated in Fig. 1.

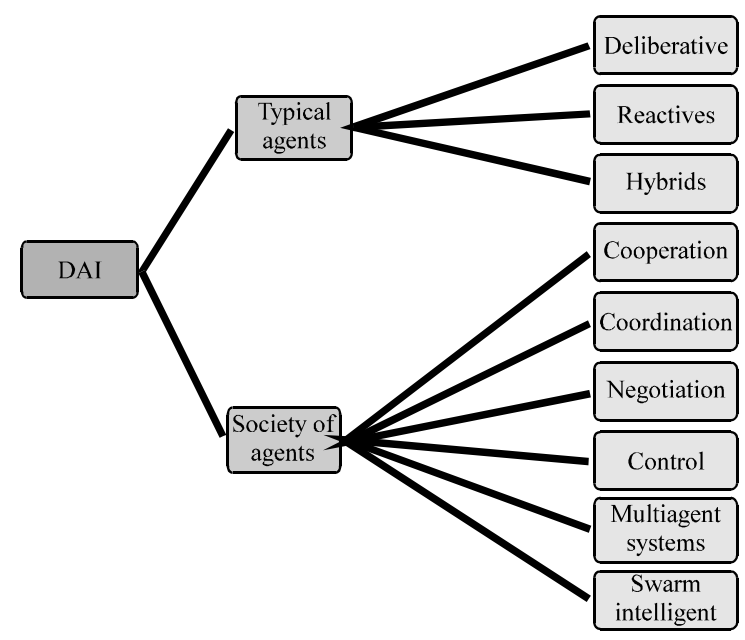

Fig. 1: Issues related to distributed artificial intelligence (Liu and $\mathrm{Wu}, 2001$ ) 


\section{Advantages:}

- Allows the addition of new agents

- Specialize in a specific task

- Better response times

- Reduce complexity as it performs cooperative tasks

- The solution of one agent is incorporated into another

\section{Study problems:}

- How to train agents to communicate and interact

- How to ensure that agents act coherently when making decisions or taking actions, accommodating the global effects of local decisions and preventing unwanted interactions

- How to train agents to represent and reason about actions, plans and knowledge of other agents and to coordinate them

- How to recognize and reconcile points of view and conflicting intentions among a set of agents to coordinate actions

Typical agents: It is understood as a methodology or architecture to build agents which means that the agent can be broken down into a set of components that can interact and provide a response, based on the following schemes.

Deliberative: This type of agent focuses on autonomy and cooperation for the execution of tasks, depending on precise models and predictability. The architecture of deliberative control (Fig. 2) is based on biological and psychological models to explain intelligent behavior, able to be used in complex algorithms providing optimal solutions which usually causes slowness in the response of the system. Its operation is done by means of the perception with the input values of the sensors which reason the sensory information and execute it by transforming it into activation commands.

Reactive: This type of agent receives the characteristics of the environment (stimuli) which allow it to develop a direct and rapid response this thanks to the division of tasks the agent is able to see itself as a grouping of modules that act independently to accomplish certain tasks (Stuart and Norving, 1995).

The reactive control architecture is based on biological models that seek to explain the behavior observed in different living organisms and also implement control strategies that respond to a collection of "conditions-actions". For example, the response to a stimulus is of a reflexive type in which the movements of the robot are made only from the information that is

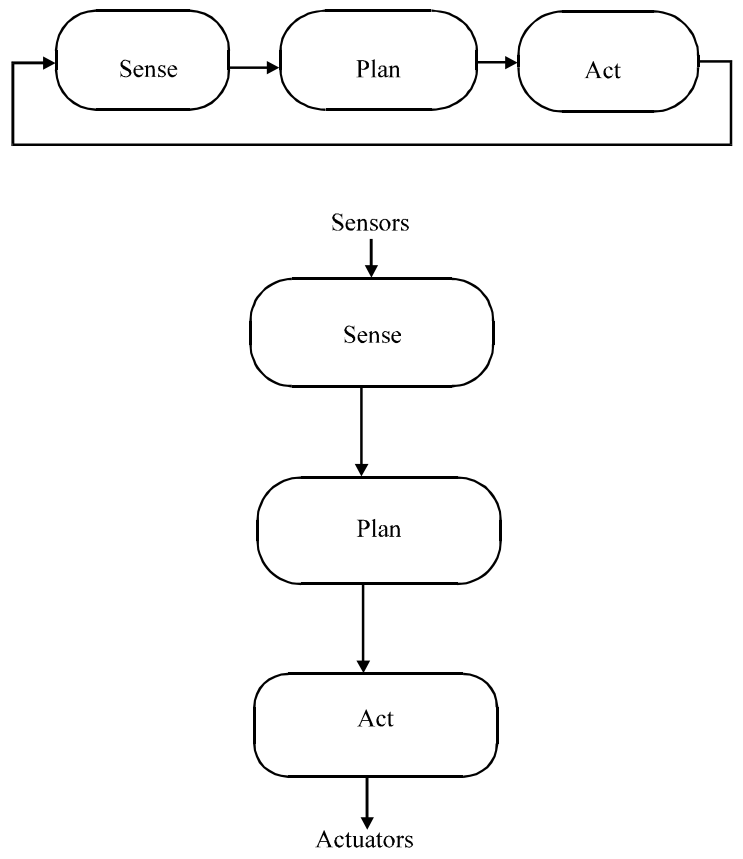

Fig. 2: Deliberative control architecture

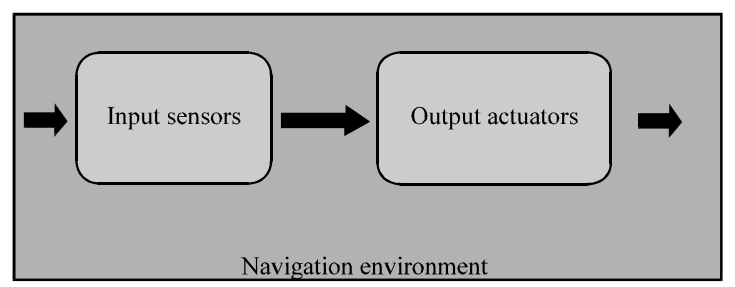

Fig. 3: Reactive control architecture

captured in the sensors (Hossian et al., 2015) from which it can be inferred that the robot perceives the world and executes the action that seems most convenient (Fig. 3).

Hybrids: it is the combination of deliberative and reactive taking the advantages offered by each agent. Its architecture is based on a SPA cycle (Fig. 4) where in the planning stage, the actions of the robot are plotted according to the global objectives and with sequences of base actions executed by modules programmed in the reactive architecture for which the speed of execution under real-time systems is the fundamental basis to which the level of reasoning of the deliberative model is integrated.

According to, its scheme in the first stage where it is raised the best way to divide the task to be carried out in several sub-tasks the appropriate behaviors to reach his objective under sensory information are selected which 


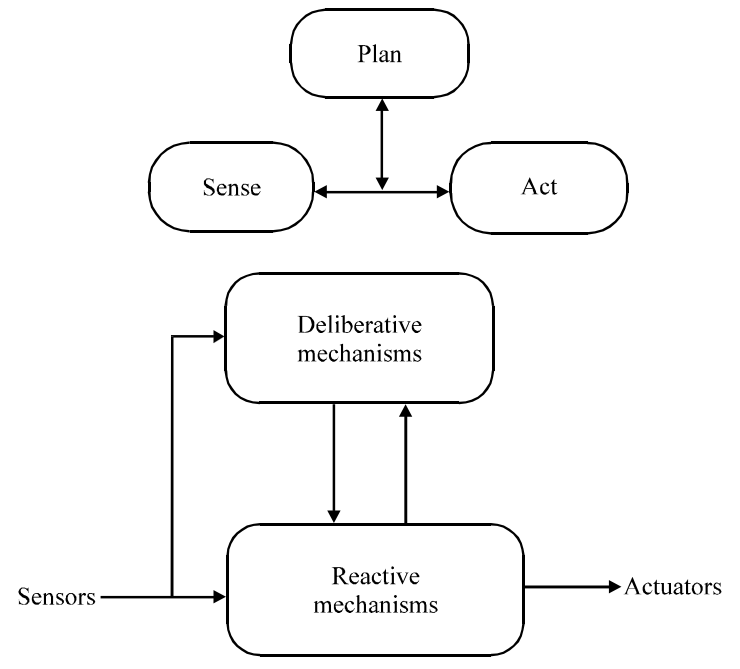

Fig. 4: Hybrid control architecture

passes to a second stage where the perception and execution of the behaviors where the values of the sensors that perform the action commands are executed.

\section{Society of agents}

Cooperation: It is a mechanism based on efficiency to cooperate where each agent must communicate in some way with others, integrate information from their environment and its neighbors to form a global understanding of the environment.

Coordination: it is a mechanism where the component of a system is arranged methodically to join efforts and contribute to an objective this allows to consider the tasks that must be perform so as not to execute actions that are not necessary. Coordination is an important mechanism that involves the synchronization of actions and the exchange of information that allow knowing and perceiving the behavior of agents, exchanging results that contribute to a general task.

Control: It is a mechanism that provides support in the implementation of coordination mechanisms in multi-agent systems. The control is related to determine the most important sub-tasks to perform in a moment and estimates the generation time of the solution.

Multiagent robotic systems: The Multiagent Robotic Systems (MARS) are based on the study of algorithms for the reception, cognition and behavior of systems composed of a group of robotic agents (Veloso and Nardi, 2006) where it must contain at least one autonomous agent. This system is characterized by having individual

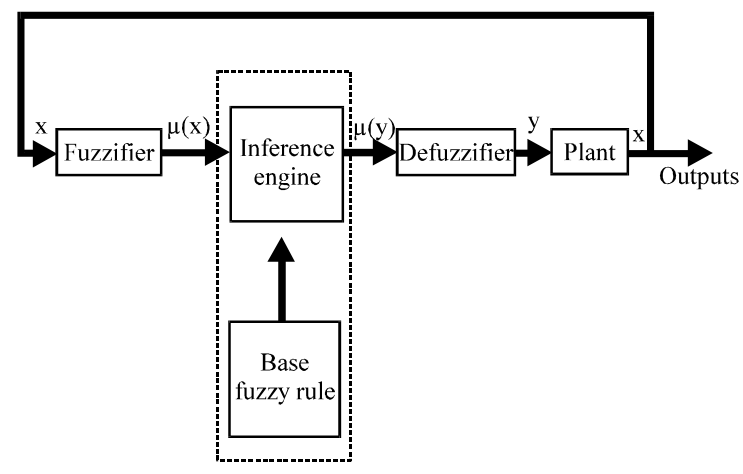

Fig. 5: Fuzzy logic controller architecture

complexity in some agents since they have global sensory information, perform complex actions, localization capacity, learning capacity, group knowledge and objective, execute assigned tasks in a synchronous or sequential manner.

\section{Learning methods for a robotic agent}

Fuzzy logic: By Zadeh (1965) carried out the first investigations in the field of fuzzy logic, establishing a means to represent and manipulate non-precise information. This technique is based on the theory of fuzzy sets which is implemented for the management of uncertain, imprecise or unmodeled data in intelligent decision systems but to determine the degree of membership of an object in a fuzzy set within the interval of the unit what has been a point in a set partially ordered under the theory of sets (Tunstel et al., 1996) cognitive relations are established by linguistic terms.

The general proposal of diffuse modeling starts from the stages of reasoning for the inference process which are presented by Wang and $\mathrm{Xu}$ (2003). Its development in robotic systems focuses on motion control systems capable of transforming a linguistic control strategy, based on the knowledge of an expert into an automatic control strategy, developed under simple fuzzy rules that are designed to make intelligent decisions in real time that allow robot movement (Santos and Duro, 2005) this type of rules of various degrees and multiple actions are combined in a result which will be interpolated.

The architecture of a fuzzy logic controller (Fig. 5) is based on an architecture that receives its inputs through a fuzzifier which is in charge of transforming the discrete values into fuzzy terms belonging to a set that is also of fuzzy nature. This is done through the membership functions based on linear mathematical models which are responsible for indicating the membership of a set and the relations between input and output sets by means of fuzzy rules of the antecedent-consequent (if-then) type, thus, determining through the reverse process of 
fuzzification (Defuzzifier) the output of the controller. The mathematical methods to perform this inverse assignment are centroid, maximum average, weighted average and membership of the maximum mean.

Neural networks (backpropagation algorithm): It is a supervised learning technique that consists of a three-layer main structure; Input, hidden and output (Fig. 6) which seeks to adjust the weights of each layer that determine learning. Said calculation is made from a set of training data with a known input/output ratio, making the activation of the layers by means of a linear function or not (for example, sigmoid). Backpropagation consists of making a comparison between the current output and the desired output by calculating the error with which the weights in each layer will be adjusted and iterating this process until it is reduced. This training is applied in order to observe its behavior against any test pattern that does not contain exactly the same input parameters with which it was trained.

This algorithm is used for reactive control in areas of autonomous navigation in robotics (Santos and Duro, 2005) it is also known as an approach based on behaviors to which a robotic system reacts, determined by the states of the sensors and performing calculations to decide the action that is going to be executed (Baturone, 2007).

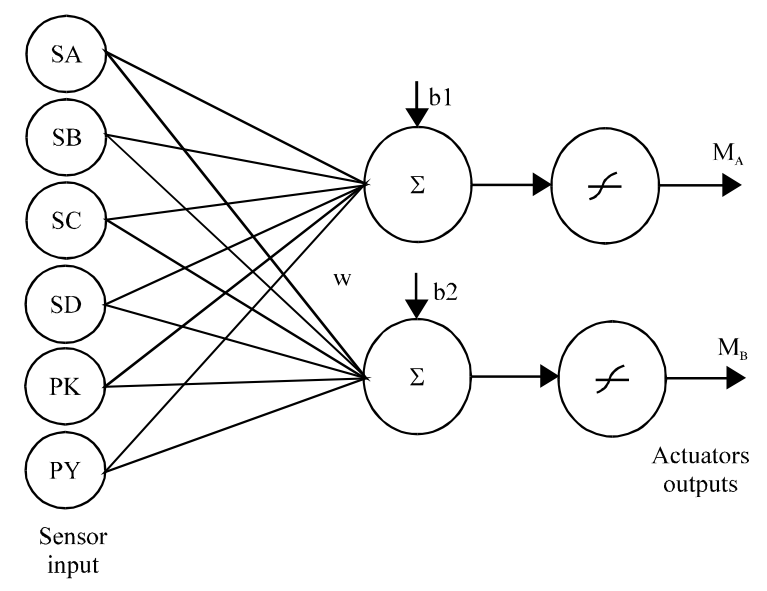

Fig. 6: Backpropagation neural network model (Hossian et al., 2015)
Deep convolutional neural networks: Convolutional neuronal networks are defined as trainable structures composed of several stages where each stage is composed of a convolution layer, activation function and subsampling layer (Fig. 7).

The convolution layer is responsible for receiving the input data and apply one or several filters which set the number of feature maps that are formed these filters are moved to a step or stride which stipulates the number of specific characteristics that are extracted from the input data, the smaller its step the greater the number of characteristics. After this it is passes through a non-linear activation function in charge of replacing the negative values in the feature map with zeros, increasing the nonlinear properties of the model which is followed by the sub-sampling layer which uses sampling operations that reduce the spatial dimensions of the input data, allowing the following convolution layers to extract better features. This technique is oriented to the recognition of patterns in images which allows the development of robotic applications based on the interaction with the environment through the capture of images.

Q-learning (learning by reinforcement): It is a reinforcement learning model that allows the resolution of sequential decision problems, i.e., the action depends on a sequence of decisions and the state in which the agent is located. It is based on the mathematical Markov Decision Processes (MDP) Model which allows to define in a precise way the task to be solved and the solution form. In general, the reward stimulus is the main idea within this model, the agent must be connected to an environment (place where the task is performed) which will be the way of perception and action to make the best solution to the problem, under which it will be rewarded.

In the model presented in Fig. 8, it can be observed that the agent receives as input the current state in which it is located and it responds with an action that is available in the database which allows it to know the state in a time interval $(\mathrm{t})$. This action generated by the agent makes a change of state to a state following the one that was in the environment, taking into account that the next state will be reported and an immediate reward signal will

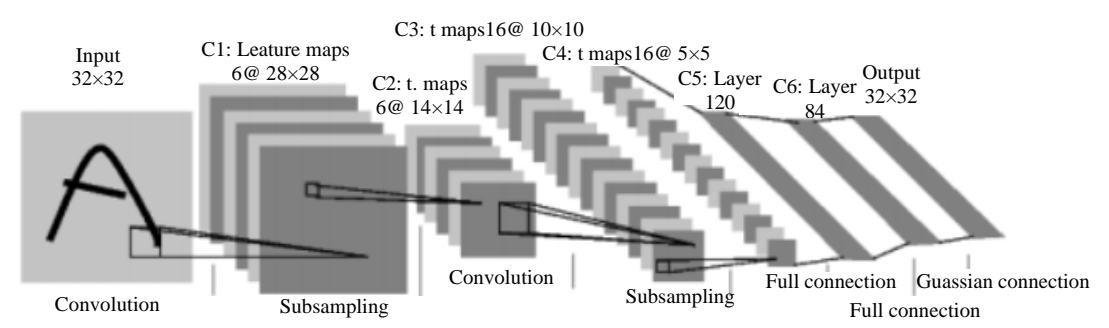

Fig. 7: Convolutional neural network architecture (Krizhesvsky, 2014) 


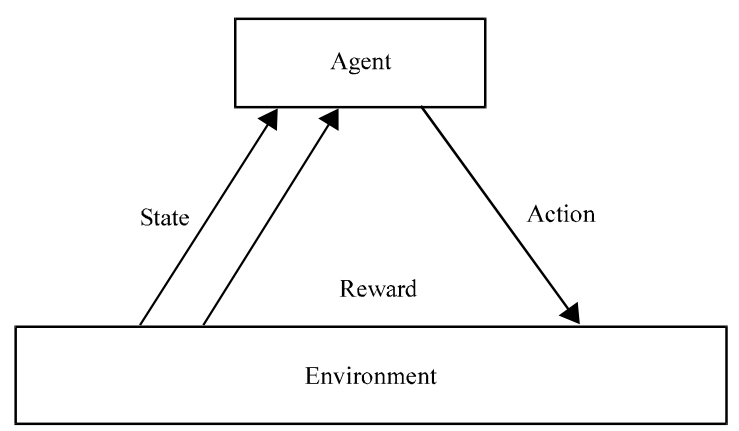

Fig. 8: Learning by reinforcement

be added for the action that was taken previously. These rules of immediate reward are defined previously in a function governed by the objectives of the agent, finding a behavior that allows it to decide in each state the actions that maximize the value of rewarding over time.

The algorithm needs to keep a record of the rewards that it received with the state and action that it took this table is known as a function $(Q)$ in which 2 parameters must be adjusted that are the learning speed which determines how much can be learned in each experience and the discount factor that determines how important the reinforcements are.

Related work in the area: An intelligent robot is that agent capable of interacting with its environment, based on the decision making derived from the information captured by its sensors, processed by its computer system and which are executed by its actuators, affecting to some extent this environment. From the above some research are presented that perform the integration of robotic devices with artificial intelligence.

In the area of autonomous navigation for mobile robots by a development through neural networks is presented where the goal of achieving autonomous navigation of robots in their area of interaction, including different obstacles is presented. In this way it is presented a navigation strategy based on the implementation of neural networks with supervised learning which has a multi-layer perceptron neuronal architecture thanks to its versatility in the implementation of non-linearly separable classification problems. This architecture consists of 6 inputs 4 neurons in the hidden layer and 3 in the output, all of them employ a sigmoidal-type activation function that allows the robot to perceive the environment and determine its course of action.

On this same way as a basis for the development of autonomous vehicles by Rusu et al. (2010) a reactive control system is presented under fuzzy logic to evade obstacles in a mobile robot using infrared sensors as

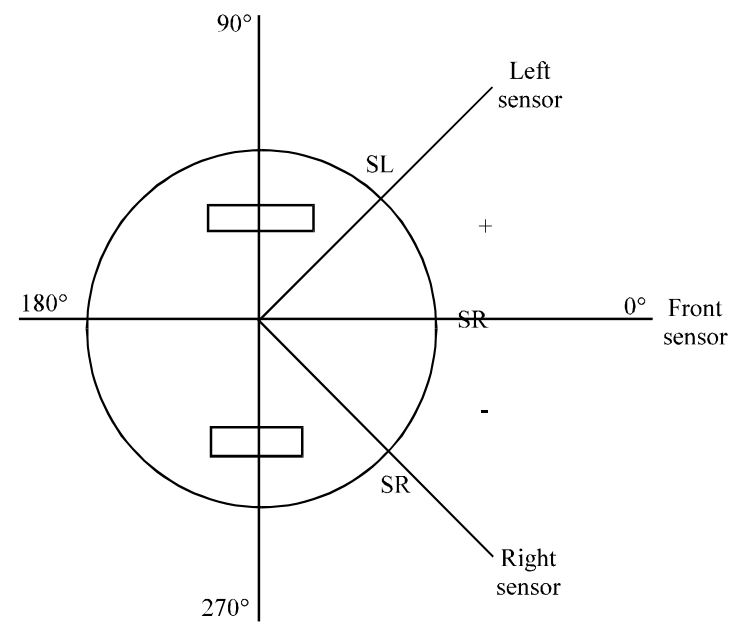

Fig. 9: Controller architecture by fuzzy logic (Rusu et al., 2010)

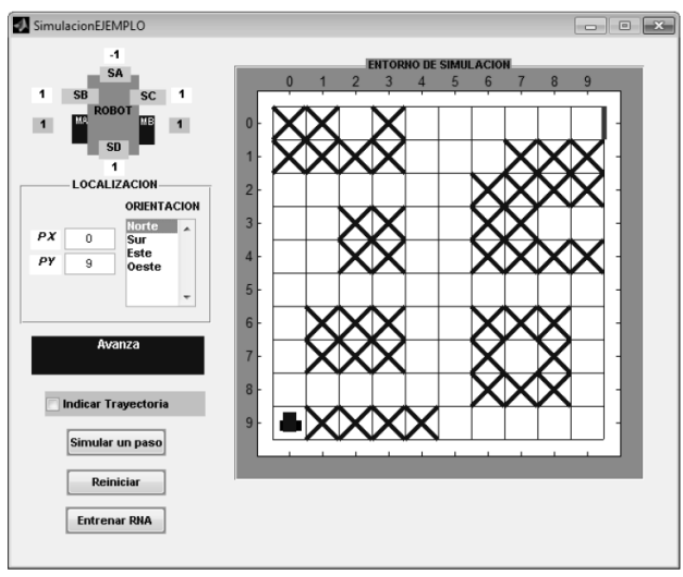

Fig. 10: Trajectory of training (Hossian et al., 2015)

shown in Fig. 9 looking for a collision-free navigation by means of 2 independent motors for its mobilization. To avoid obstacles without colliding with any of these the mobile robot acquires the sensory information of 3 possible sources (left sensor, front sensor and right sensor) by means of triangular membership functions with respect to the distance (near, middle and far) and defuzzified output is taken as response that controls the speed of the motors.

Another implementation of an algorithm based on artificial backpropagation neural networks is presented by Hossain et al. (2015) which seeks to evaluate the performance of a mobile robot in a computerized simulation environment and under reactive control premises. The environment is designed with a graphical interface as shown in Fig. 10 which allows locating the obstacles, initial and final position of the robot it has a 


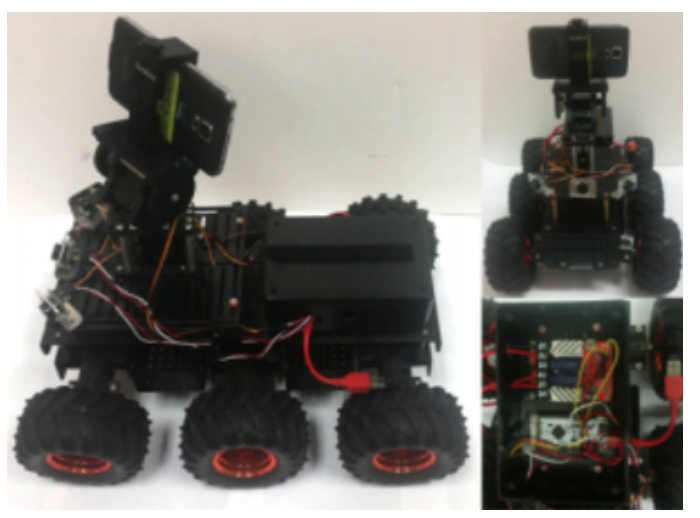

Fig. 11: Autonomous vehicle based on android robotics (Hwu et al., 2017)

button to initialize the training to the different input values. It was implemented an algorithm under a three-layer architecture the input with 6 neurons (sensors and positions $(\mathrm{x}, \mathrm{y})$ ) a hidden layer with 2 neurons and the output layer with 2 neurons (motors). This architecture is trained from the development of a sensor-motor map that includes the trajectory to which the training is applied, determining the best navigation scheme in response to obstacles and initial/final points that were entered.

By Hwu et al. (2017) they use the neo-morphic computation method considered as the solution to reduce size, weight and power in mobile embedded systems where they implement the first communication system powered by batteries of IBM TrueNorth NSle and the autonomous platform based on AndroidbRobotics which is seen in Fig. 11. In that research they build a trajectory behavior data set by manually driving the robot along mountain trails and recording video frames, generating a database for the training of a deep Convolutional Neuronal Network (CNN) which translates into input trigger patterns on the energy efficiency platform (TrueNorth NS1e) reaching an accuracy of $90 \%$ that was obtained after 10,000 iterations and a total success in the self-driving of the robot when crossing a steep path of the mountain.

On the other hand in the field of robotic manipulators is the work presented by Lenze et al. (2015) where a solution to the problem of grip of a robot arm that detects objects in RGB-D scale is proposed. The solution is oriented to Baxter and PR2 robots, implementing the deep learning approach under 2 cascade systems with 2 deep neural networks where the main detections are reevaluated by the second network which means that in the first network there are fewer features, making it faster in execution, performing a data debugging of which are

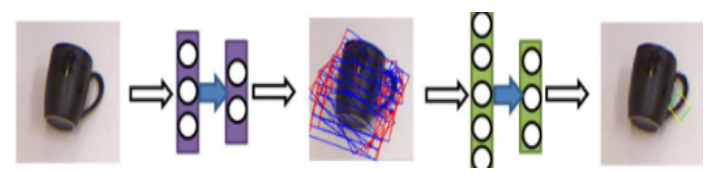

Fig. 12: 2-Stage detection process (Lenz et al., 2015)

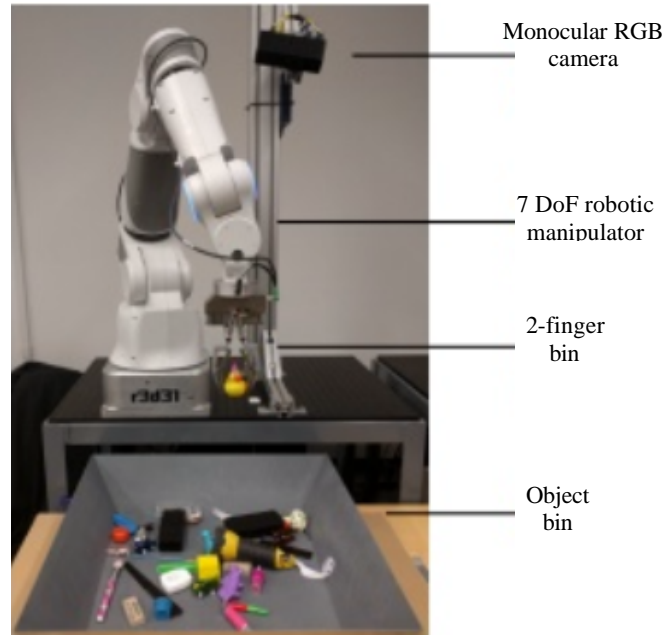

Fig. 13: Robotic manipulator implemented for data collection (Levine et al., 2016)

unlikely grip of the robot and in the second network that has more parameters, so, it is deeper is used to determine the rectangle that tops the list of candidates for the best grip as can be seen in Fig. 12. The robotic platform Baxter showed a success rate of $84 \%$ and the PR2 robot of $89 \%$ at the time of execution of a varied grip of a set of objects for it it was built a database containing more than 1035 images of 280 gripping objects.

By Lavine et al. (2016) it is presented a project oriented to predict the probability of spatial movements of the robotic arm end effector by means of the images captured with a monocular camera where the application of the convolutional network used is oriented to be able to observe the relationship between the gripper and the objects of the scene. To fulfill the aforementioned task, a depth learning was implemented by means of a CNN, collecting a total of 800,000 grasping attempts over a period of 2 months to train the prediction model of a robotic arm for this validations were made between 6-14 robotic manipulator arms with the architecture shown in Fig. 13 and 14 which is developed under different spatial scenarios where the position of the camera with respect to the manipulator is highlighted. From the above, the network takes a picture that is considered as the entrance and at the same time as an additional image that is being registered before the gripper begins its journey. In 

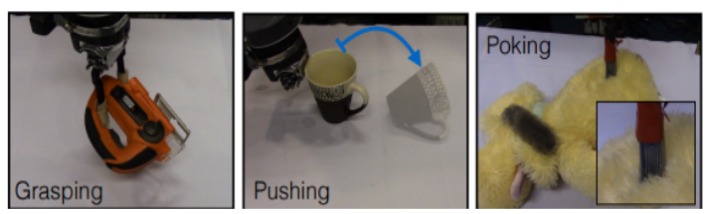

Fig. 14: Robot manipulator for gripping, pushing and touching objects (Pinto et al., 2016)

addition, a CNN is used to continuously update the commands of the robot's motor always choosing the best path to success these latter mechanisms give it the name of servoing GRASP which provides the robot with a fast feedback to disturbances and movements of objects (Lavine et al., 2016).

Likewise by Pinto et al. (2016), it is presented the construction of a Baxter platform composed of a parallel jaw gripper and a skin with tactile sensors that interacts with the world. By training convolutional networks the platform is able to develop activities such as pushing, touching (Haptic data) and gripping the objects that has in its environment as shown in Fig. 13 snd 14. With these 3 different physical interactions it collects about 130,000 data that come from the $\mathrm{CNN}$ architecture trained which supervised these interactions, showing the quality of the representations that the neuron learned by observing and performing the activations with its closest neighbor in the instance recovery task.

By Nair et al. (2017) a system is presented where a robot (Baxter) is trained by flexible learning for the manipulation of deformable objects such as a rope where the robot uses a model based on an inverse dynamic that is learned to execute trajectory tracking actions. For this, a convolutional neuronal network is used that consists of 6 convolution layers, the first with 96 filters, the second with 256 filters, the third with 384 filters, the fourth with 384 filters, the fifth with 256 filters and the last with 500 filters and which will connect to 2 fully connected layers of 200 units which allows the robot to learn the inverse model by self-supervision which finally enables the robot to perform 60,000 interactions with the rope without human supervision, thus, forming a predictive model of behavior. The aforementioned architecture consists of 2 parts based on the capture of an image at a time ( $\mathrm{t}$ ) and then a second capture of the image one at time $(\mathrm{t}+1)$ where the output of this network is the final action of the robot that moves the rope as seen in the Fig. 15.

Among the most recent developments in the field of intelligent robots, Kumar et al. (2017) present an automated robotic agent for the task of collecting and storing objects. This is developed in four stages. First, there is the perception moduleresponsible for recognizing

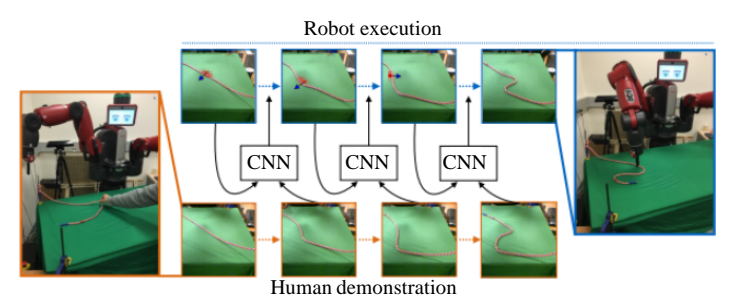

Fig. 15: Demonstration of human configurations and execution performed by the robot through learning by CNN (Nair et al., 2017)

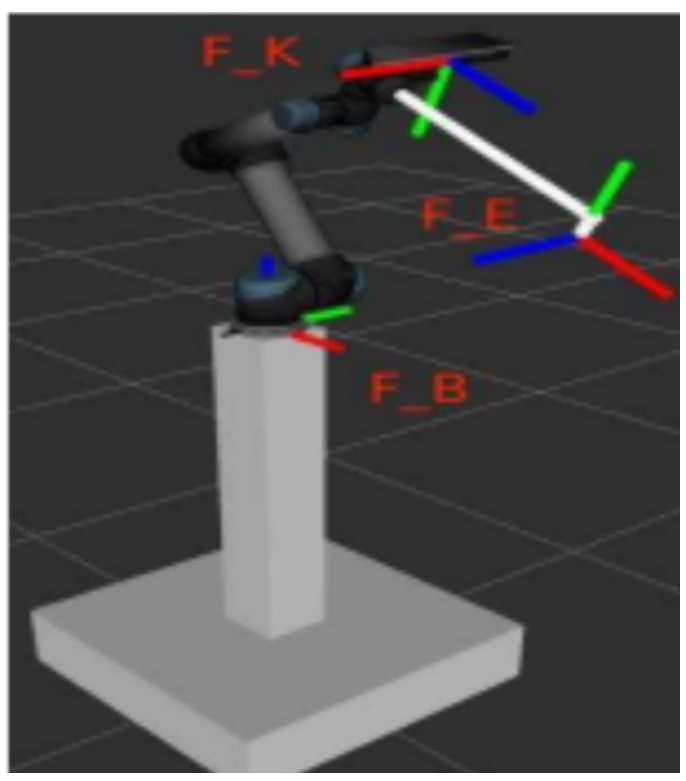

Fig. 16: Coordinate system for the robot performing the calibration (Kumar et al., 2017)

objects in a 3D space through a convolutional neuronal network. Second, the planning module responsible for generating the routes, so that, the gripper or end effector reaches the objects. Third, the calibration module responsible for defining the physical space for the robot through a vision system. Finally, the aspiration system to collect an object. The simulation architecture used can be seen in Fig. 16.

The database for object recognition was built from 150 images by object to be detected where different orientations, lighting and different physical environments are used to expand the information on each object from this detailed database a total of 6,000 images of 40 different objects were acquired. The architecture of the network was formed with 13 convolutional layers and 3 fully connected layers. This algorithm presented a recognition accuracy of $90 \%$ for 29 objects with an input of 4,800 training samples. 


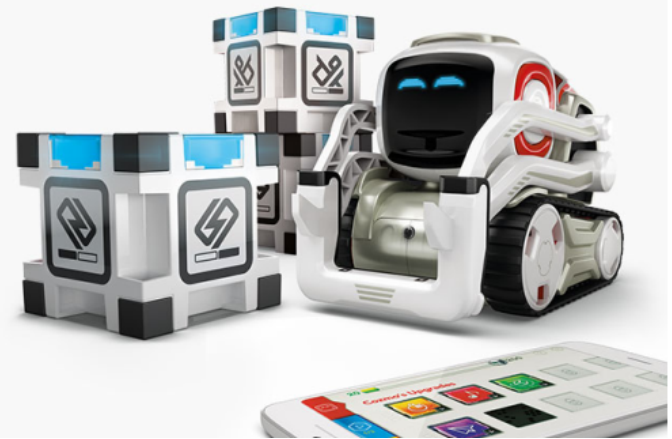

Fig. 17: Cozmo robot (Anonynous, 2017)

Featured developments: Now a days it has been established that a robot designed under the artificial intelligence architecture is able to reach the intellectual coefficient of a 4 years old child what leads to think that in a short time a robot could occupy jobs where now managers are human, equaling or exceeding the intellectual capacity required in any work. The apogee of this new technology based on artificial intelligence has captured the interest of researchers for example the scientists of the technological institute of Tokyo launched a robot capable of learning from its environment, deducting the action it should do based on the acquired learning but if it does not have the knowledge or the ability to solve its problem it can connect to the internet and ask other robots what it can do. This is thanks to a programming algorithm called Self Organizing Incremental Neural Network (SOINN) this type of architecture is adapted to supervised, semi-supervised and unsupervised learning, operating incrementally which means that it has the capacity to learn from past experiences without destroying what has been acquired (Shen and Hasegawa, 2010). Derived from the technological advances in this field, some more outstanding commercial applications are presented below that have been originated thanks to the union of these two technologies, robotics and artificial intelligence as a sample of the potential that they project towards the future.

Cozmo: As shown in Fig. 17 this is a robotic design by the company Anki to pretend that it has "soul" whose goal is to create an emotional bond with the owner. It is equipped with a camera and multiple sensors which allow to integrate a zone mapping to recognize where it is and avoid falls it is able to move and play thanks to its system designed under artificial intelligence that allows it to recognize faces and voices, recreating emotions of fear and distrust when he meets a new person or to cry when it loses a game (Anonymous, 2017).

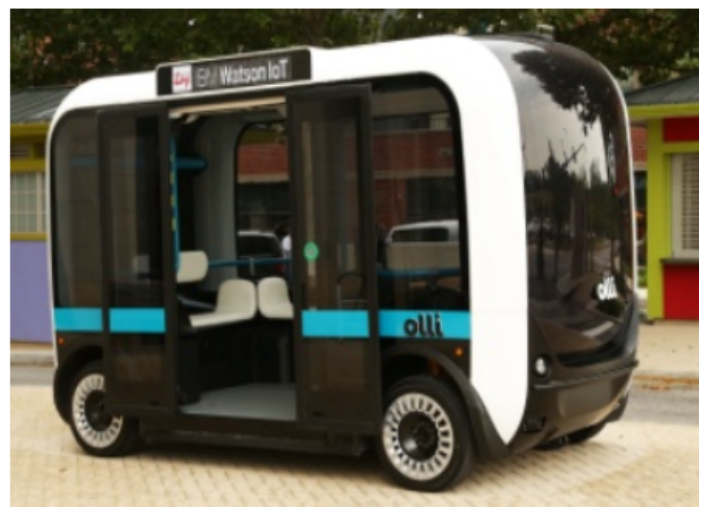

Fig. 18: Automnomous vehicle olli (Anonymous, 2017)

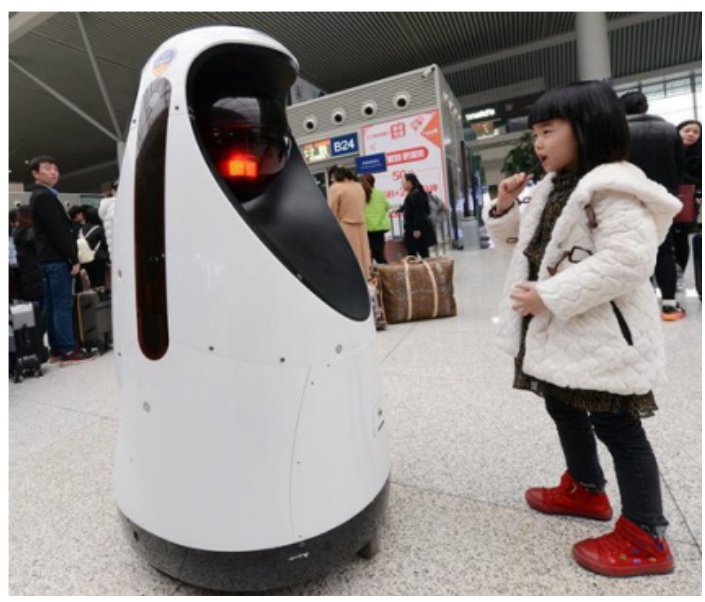

Fig. 19: E-patrol Sheriff (Anonymous, 2017)

Olli: Designed by the IBM company (Fig. 18). It incorporates the IBM Watson Explorer platform developed under artificial intelligence and is capable of converting voice to text and classification of natural language which means that it is capable of processing a chaste language, allows the extraction of entities, text to speech conversion and thanks to its development in access and search on the web it provides the ability to answer questions about restaurants or historical places, due to the combination of these techniques the vehicle is able to receive orders such as "take me to the city center" (Anonymous, 2017).

E-patrol: or Robot Sheriff is a robotic agent that patrols Ten Station (China) in search of criminals as shown in Fig. 19 with the ability to detect fires, moves autonomously and is equipped by a camera system. It is also capable of measuring the levels of air quality and temperature, through integrated technology based on artificial intelligence, more specifically on neural networks 


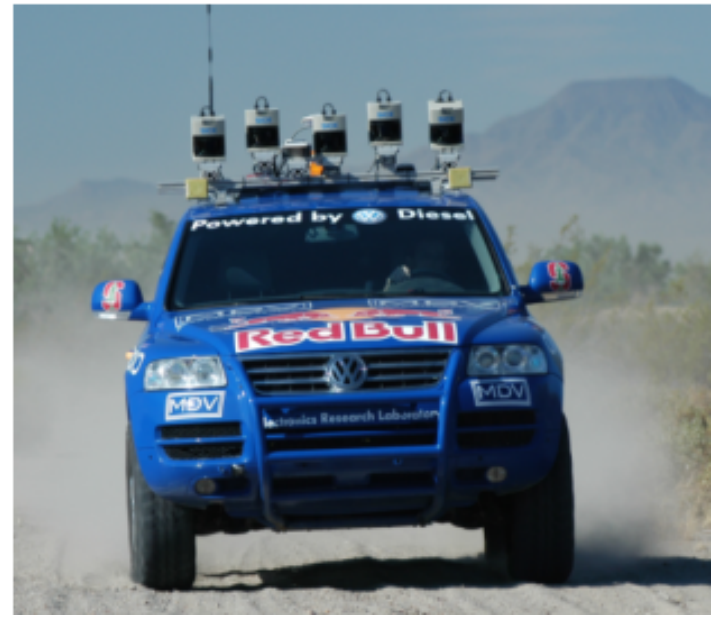

Fig. 20: Autonomous vehicle stanley (Thurn et al., 2006)

that support facial recognition which contains the database of criminals and most wanted people in this country (Anonymous, 2017).

Stanley: "The robot that won the DAPRA Grand Challenge" (Thrun et al., 2006) is an autonomous vehicle developed by the racing team of Stanford University and associates as shown in Fig. 20. It is based on software developed under artificial intelligence techniques capable of processing the data obtained by its sensors to determine the proper direction, acceleration, brake and change of direction in turn allows it to coordinate the ascension by means of a data-based scoring function which modifies each parameter one by one, determining if the new value is an improvement of the previous one evaluated through a data record and if it is correct is retained or if an improvement is not found during a certain interval, the search interval makes a cut in its data only leaving half of the information and resuming the search. In this way, a probabilistic analysis is applied which has the effect of determining the accuracy of the terrain labels, generating a map sufficient to drive. This algorithm is implemented before and during a race which makes the information available to the vehicle robust and accurate (Thrun et al., 2006).

Mapgen: Thruns et al. (2006) is a remote agent that combines 2 technologies the APGEN that is an activity planning tool and the European Planning/Scheduling System, taking advantage of each one's strengths, allowing it to be able to build a viable tactical planning, flight planning and even conflict resolution. The user has the degree of authority to make the input adjustments in order to produce a superior plan and thus the MPAGEN is able to provide the ability to exercise finer control to facilitate modes of manual operation, performs daily operation plans for Mars exploration vehicles at NASA (Ai-Chang et al., 2004).

\section{CONCLUSION}

The evolution of artificial intelligence in robotics is one of the fields that has aroused greater interest in the industry from fuzzy control to control based on deep learning with Research Journal of Applied Sciences 12 (3): convolutional neural networks. As shown previously it is a science that is in development and its integration with robotics has generated the question whether it would be possible to develop a homo sapiens robot or better known as a human robot capable of thinking, knowing that to date there is already a robot with the intellectual capacity of a 4 years old child and a machine capable of passing the Turing test evaluated as a 13 years old adolescent, the answer will be obtained in a few years, since, the growth and technological progress in recent times has had an exponential behavior.

The application of intelligent algorithms based on convolutional neural networks in the field of robotics has helped to create robotic agents where manipulative robots stand out as object classifiers, mobile robots in trajectory planning optimizing response times with the highest precision and avoiding obstacles with the object recognition algorithm, police robots that capture fugitives from justice or seek missing persons or as entertainment for the little ones, a friend with whom they can interact and be able to transmit a feeling with only one glance, this is the technology with which the current world develops.

The development of robotics and artificial intelligence is expected to support sectors such as security, mitigating the risk of human losses, health by supporting physicians with assistants capable of performing tasks with much more precision and without fear of being wrong.

\section{ACKNOWLEDGEMENTS}

The researchers are grateful to the Nueva Granada Military University which through its Vice chancellor for research, finances the present project with code IMP-ING-2290 and titled "Prototype of robot assistance for surgery" from which the present work is derived.

\section{REFERENCES}

Ai-Chang, M., J. Bresina, L. Charest, A. Chase and J.J. Hsu et al., 2004. Mapgen: Mixed-initiative planning and scheduling for the mars exploration rover mission. IEEE. Intell. Syst., 19: 8-12. 
Anonymous, 2017. China's E-patrol robot sheriff tracks down criminals through facial recognition. Latin Post, New York, USA.

Anonymous, 2017. Meet Olli. Local Motors, Phoenix, Arizona. https://localmotors.com/meet-olli/

Anonymous, 2017. The little bot with super-powered smarts. ANKI, San Francisco, California.

Baturone, A.O., 2007. [Robotics Manipulators and Mobile Robots]. Ed. Alfaomega, Barcelona, Spain, (In Spanish).

Carolina, O. and C. Lemus, 2011. [Design of a Model Based on Artificial Intelligence Techniques for the Development of an Intelligent Learning-Oriented System]. ITCA Publisher, Santa Tecla, El Salvador, ISBN:978-99923-988-3-8, (In Spanish).

Hossian, A., L. Cejas, R.C. Echeverria, V. Olivera and M. Alveal, 2015. [Development of experiments of a navigating robot with neural networks in a structured environment: Programming behaviors with retropropagation algorithm (In Spanish)]. Latin Am. J. Software Eng., 3: 41-46.

Hwu, T., J. Isbell, N. Oros and J. Krichmar, 2017. A self-driving robot using deep convolutional neural networks on neuromorphic hardware. Proceedings of the International Joint Conference on Neural Networks (IJCNN'17), May 14-19, 2017, IEEE, Anchorage, Alaska, USA., ISBN:978-1-5090-6183-9, pp: 635-641.

Krizhevsky, A., 2014. One weird trick for parallelizing convolutional neural networks. Neural Evol. Comput., 1: $1-7$.

Krizhevsky, A., I. Sutskever and G.E. Hinton, 2012. Imagenet Classification with Deep Convolutional Neural Networks. In: Advances in Neural Information Processing Systems, Leen, T.K., G.D. Thomas and T. Volker (Eds.). MTT Press, Cambridge, Massachusetts, USA., ISBN:0-262-12241-3, pp: 1097-1105.

Kumar, S., A. Majumder, S. Dutta, R. Raja and S. Jotawar et al., 2017. Design and development of an automated robotic pick and stow system for an E-commerce warehouse. Rob., 1: 1-15.

Lenz, I., H. Lee and A. Saxena, 2015. Deep learning for detecting robotic grasps. Intl. J. Rob. Res., 34: 705-724.

Levine, S., P. Pastor, A. Krizhevsky and D. Quillen, 2016. Learning Hand-Eye Coordination for Robotic Grasping with Large-Scale Data Collection. In: International Symposium on Experimental Robotics, Springer, Cham, Switzerland, ISBN:978-3-319-50114-7, pp: $173-184$.

Liu, J. and J. Wu, 2001. Multiagent Robotic Systems. CRC Press, Boca Raton, Florida, USA., Pages: 301.
McClelland, J. and D. Rumelhart, 1986. Explorations in Parallel Distributed Processing, a Handbook of Models, Programs and Exercises. The MIT Press, Cambridge, Massachusetts,

McCulloch, W.S. and W. Pitts, 1943. A logical calculus of the ideas immanent in nervous activity. Bull. Math. Biophys., 5: 115-133.

Nair, A., D. Chen, P. Agrawal, P. Isola and P. Abbeel et al., 2017. Combining self-supervised learning and imitation for vision-based rope manipulation. Proceedings of the IEEE International Conference on Robotics and Automation (ICRA'17), May 29-June 3, 2017, IEEE, Singapore, ISBN:978-1 5090-4634-8, pp: 2146-2153.

Nonami, K., R.K. Barai, A. Irawan and M.R. Daud, 2013. Hydraulically Actuated Hexapod Robots: Design, Implementation and Control. Springer, Berlin, Germany, ISBN:978-4-431-54348-0, Pages: 271.

Pinto, L., D. Gandhi, Y. Han, Y.L. Park and A. Gupta, 2016. The Curious Robot: Learning Visual Representations via Physical Interactions. In: Computer Vision, Leibe, B., J. Matas, N. Sebe and M. Welling (Eds.). Springer, Cham, Switzerland, ISBN:978-3-319-46474-9, pp: 3-18.

Poole, D., A.K.M. Ckworth and R. Goebel, 1998. Computational Intelligence: A Logical Approach. Oxford University Press, Oxford, England, UK., ISBN:9780195102703, Pages: 558.

Rich, E. and K. Knight, 1991. Artificial Intelligence. McGraw-Hill, New York, USA.

Rusu, C.G., I.T. Birou and E. Szoke, 2010. Fuzzy based obstacle avoidance system for autonomous mobile robot. Proceedings of the IEEE International Conference on Automation Quality and Testing Robotics (AQTR'10) Vol. 1, May 28-30, 2010, IEEE, Cluj-Napoca, Romania, ISBN:978-1-4244-6724-2, pp: 1-6.

Santos, J. and R.J. Duro, 2005. [Artificial Evolution and Autonomous Robotics]. Alfaomega, Kalmar, Sweden, ISBN:9789701510957, Pages: 234 (In Spanish).

Scheinman, V.D., 1969. Design of a computer controlled manipulator. Master Thesis, Stanford University Computer Science, California.

Shen, F. and O. Hasegawa, 2010. Self-Organizing Incremental Neural Network and its Application. In: Artificial Neural Networks, Diamantaras, K., W. Duch and L.S. Iliadis (Eds.). Springer, Berlin, Germany, ISBN:978-3-642-15824-7, pp: 535-540.

Simonyan, K. and A. Zisserman, 2014. Very deep convolutional networks for large-scale image recognition. Master Thesis, Cornell University, Ithaca, New York.

Stuart, J. and P. Norving, 1995. Artificial Intelligence: A Moderm Approah. Prentice-Hall, Upper Saddle River, New Jersey, USA., ISBN:9780131038059, Pages: 932. 
Thrun, S., M. Montemerlo, H. Dahlkamp, D. Stavens and A. Aron et al., 2006. Stanley: The robot that won the DARPA grand challenge. J. Field Rob., 23: 661-692.

Tunstel, E., T. Lippincott and M. Jamshidi, 1996. Introduction to fuzzy logic control with application to mobile robotics. Master Thesis, University of New Mexico, Albuquerque, New Mexico.

Turing, A.M., 1950. Computing machinery and intelligence. Mind, 59: 433-460.
Veloso, M.M. and D. Nardi, 2006. Special issue on multirobot systems. Proc. IEEE., 94: 1253-1253.

Wang, D. and G. Xu, 2003. Full-state tracking and internal dynamics of nonholonomic wheeled mobile robots. IEEE. ASME. Trans. Mechatron., 8: 203-214.

Winston, P.H., 1992. Artificial Intelligence. 3rd Edn., Addison-Wesley, Boston, Massachusetts, .

Zadeh, L.A., 1965. Fuzzy sets. Inform. Control, 8: 338-353. 